\title{
Prevalência da AIDS em idosos no centro-oeste brasileiro
}

\author{
Prevalence of aids in elderly persons in the Central west Brazilian \\ Prevalencia del SIDA en idosos em el Centro-oeste brasileño
}

Amanda Tallita da Silva ${ }^{1}$, Ana Luiza Barros Parreira ${ }^{1}$, Carolina de Araújo Machado ${ }^{1}$, Daniel Cavalcante Fonseca ${ }^{1}$, Jéssica Silva do Carmo $^{1}$, Mariana de Lima Barbosa ${ }^{1}$, Heloísa Silva Guerra ${ }^{2 *}$.

\section{RESUMO}

Objetivo: Analisar a prevalência de AIDS na população idosa do Centro-Oeste, no período entre janeiro de 2008 e dezembro de 2017 , além de fornecer dados e informações que possibilitem a elaboração de medidas de promoção da saúde que sejam efetivas para a prevenção dessa moléstia na população estudada. Métodos: Trata-se de um estudo descritivo, por meio da análise dos casos de AIDS registrados no CentroOeste brasileiro entre janeiro de 2008 e dezembro de 2017, considerando-se as variáveis: faixa etária, sexo e categoria de exposição. Os dados foram coletados na base de dados do Departamento de Informática do Sistema Único de Saúde. Resultados: Os casos de AIDS em idosos representaram 4,59\% do total na região e período analisados. O maior número de casos deu-se por relação heterossexual em homens. Conclusão: Há um número considerável de idosos sexualmente ativos, mostrando que o estigma de população assexuada precisa ser desconsiderado, e é necessário criar ações em saúde direcionadas a essa faixa etária.

Palavras-chave: HIV, Síndrome da Imunodeficiência Adquirida, Idoso, Prevalência.

\begin{abstract}
Objective: To analyze the prevalence of AIDS in the elderly population of the Center-West between January 2008 and December 2017, as well as to provide data and information that enable the development of health promotion measures that are effective for the prevention of this disease in the study population. Methods: This is a descriptive study, by means of the analysis of AIDS cases recorded in the Brazilian Midwest between January 2008 and December 2017, considering the following variables: age, sex and exposure category. The data were collected in the database of the Information System of the Unified Health System. Results: AIDS cases in the elderly represented $4.59 \%$ of the total in the region and period analyzed. The highest number of cases occurred because of heterosexual relationship in men. Conclusion: There are a considerable number of sexually active elderly, showing that the stigma of the asexual population needs to be disregarded, and it is necessary to create health actions directed to this age group.
\end{abstract}

Key words: HIV, Acquired Immunodeficiency Syndrome, Aged, Prevalence.

\section{RESUMEN}

Objetivo: Analizar la prevalencia de SIDA en la población anciana del Centro-Oeste, en el período entre enero de 2008 y diciembre de 2017, además de proporcionar datos e informaciones que posibiliten la elaboración de medidas de promoción de la salud que sean efectivas para la prevención de esa molestia en la población estudiada. Métodos: Se trata de un estudio descriptivo, a través del análisis de los casos de SIDA registrados

${ }^{1}$ Acadêmicos de Medicina, Universidade de Rio Verde, Aparecida de Goiânia, Goiás.

${ }^{2}$ Professora Adjunto I da Faculdade de Medicina da Universidade de Rio Verde, Aparecida de Goiânia, Goiás. *E-mail: heloisasguerra@gmail.com

SUBMETIDO EM: 3/2019

ACEITO EM: 4/2019

PUBLICADO EM: 5/2019

REAS/EJCH | Vol. Sup. 23 | e434 | DOI: https://doi.org/10.25248/reas.e434.2019 Página 1 de 7 
en el Centro-Oeste brasileño entre enero de 2008 y diciembre de 2017, considerando las variables: grupo de edad, sexo y categoría de exposición. Los datos fueron recolectados en la base de datos del Departamento de Informática del Sistema Único de Salud. Resultados: Los casos de SIDA en ancianos representaron el $4,59 \%$ del total en la región y período analizados. El mayor número de casos se dio por relación heterosexual en hombres. Conclusión: Hay un número considerable de ancianos sexualmente activos, mostrando que el estigma de población asexual necesita ser desconsiderado, y es necesario crear acciones en salud dirigidas a ese grupo de edad.

Palabras clave: VIH, Síndrome de Inmunodeficiencia Adquirida, Anciano, Prevalencia.

\section{INTRODUÇÃO}

O Brasil conta, hoje, com mais de 20 milhões de pessoas com idade acima de 60 anos, representando aproximadamente 10\% da população em geral, com estimativas de aumento para 30\% em 2050 (IBGE, 2011; IBGE, 2008). As alterações da composição da população lançam um desafio para a sociedade em geral, mas a principal incitação está no âmbito da saúde e de seus profissionais, envolvendo lidar com o processo de envelhecimento e com as pessoas idosas, como uma questão social (PIMENTEL CP, 2017).

Segundo o relatório de 2015 da Organização das Nações Unidas (ONU), o envelhecimento saudável é definido como o processo de desenvolvimento e manutenção da capacidade funcional que permite o bemestar em idade avançada (ONU, 2015). O envelhecimento traz modificações importantes no que se refere aos aspectos físicos e emocionais das pessoas, porém os sentimentos e as sensações não sofrem deterioração, podendo a sexualidade ser vivenciada até o fim da vida (LAURENTINO NRS, et al., 2006).

O aumento da qualidade de vida aliado aos avanços tecnológicos em saúde, como os tratamentos de reposição hormonal e medicações que facilitam o desempenho sexual, como o sildenafil, têm permitido a persistência de práticas sexuais entre os idosos (LAROQUE MF, et al., 2011; MASCHIO MBM, et al., 2011).

Apesar da terceira idade se beneficiar atualmente com os avanços científicos e tecnológicos prolongando a sua sexualidade, são crescentes também as preocupações com as Infecções Sexualmente Transmissíveis (IST) nessa faixa etária (BRASIL, 2010). Pesquisas apontam que os idosos mantêm a vida sexual ativa e estão expostos a essas enfermidades, em especial ao vírus da imunodeficiência humana (HIV) (BRASIL, 2015; LINDAU ST, et al., 2007; NGUYEN N, et al., 2008).

Desde 1986, com a criação do Programa Nacional de DST/AIDS, o Brasil tem desenvolvido estratégias para a prevenção das IST, entretanto, muito pouco se fez em relação à população de idosos. A escassez de estudos epidemiológicos e campanhas de prevenção, somados à ampliação do período sexual ativo, processos fisiológicos do envelhecimento e aspectos comportamentais, têm refletido na incidência de IST e Síndrome da Imunodeficiência Adquirida (AIDS) nos idosos (DORNELAS NETO J, et al., 2015).

Segundo o Sistema de Informação de Agravos de Notificação (SINAN) entre 2014 e 2017 foram notificados 2.056 casos de AIDS em idosos acima de 65 anos no Brasil; tornando-se importante a realização de pesquisas que contemplem essa temática (BRASIL, 2019).

A AIDS é a uma doença causada pelo Vírus da Imunodeficiência Humana (HIV) que acomete o sistema imunológico destruindo as células de defesa do organismo (BRASIL, 2013). Em idosos, a fragilidade do sistema imunológico dificulta o diagnóstico, podendo os sintomas da infecção por HIV serem confundidos com outras infecções. O diagnóstico tardio favorece o agravamento do quadro, que pode comprometer inclusive a saúde mental dos idosos (BRASIL, 2018).

Nesse sentido, o objetivo deste estudo foi analisar a prevalência de AIDS na população idosa do CentroOeste, no período entre janeiro de 2008 e dezembro de 2017, além de fornecer dados e informações que possibilitem a elaboração de medidas de promoção da saúde que sejam efetivas para a prevenção dessa moléstia na população estudada.

REAS/EJCH | Vol. Sup. 23 | e434 | DOI: https://doi.org/10.25248/reas.e434.2019 Página 2 de 7 


\section{MÉTODOS}

Trata-se de um estudo descritivo utilizando-se dados secundários, obtidos no Sistema de Informação de Agravos de Notificação (SINAN), Sistema de Controle de Exames Laboratoriais da Rede Nacional de Contagem de Linfócitos CD4+/CD8 e Carga Viral (SISCEL) e Sistema de Informações de Mortalidade (SIM), todos inseridos na base da dados do Departamento de Informática do Sistema Único de Saúde (DATASUS).

A população do estudo foi constituída pelos casos de AIDS notificados na região Centro-Oeste brasileira, no período entre janeiro de 2008 e dezembro de 2017, considerando-se as seguintes variáveis: faixa etária (menores de 60 anos, 60 a 69 anos, 70 a 79 anos, 80 anos e mais e ignorado), sexo (masculino, feminino) e categoria de exposição hierarquizada (homossexual, bissexual, heterossexual, uso compartilhado de drogas injetáveis, hemofílico, transfusão, transmissão vertical e ignorado).

A coleta dos dados foi realizada em maio de 2018. Os dados foram inseridos em planilhas do programa Microsoft $\circledast$ Excel $\AA^{\circledR} 2016$ e analisados descritivamente, com apresentação da frequência absoluta e porcentual das variáveis por meio de tabelas. Por se tratar de dados secundários e de domínio público, não foi necessária a submissão ao Comitê de Ética em Pesquisa.

\section{RESULTADOS}

Entre 2008 e 2017 foram notificados 25.791 novos casos de AIDS na região Centro-oeste do Brasil. Destes, 1.184 foram relativos à população idosa, representando $4,59 \%$ do número total de casos (Tabela 1). Em indivíduos menores que 60 anos se apresentou um aumento no número de casos no período de 2008 a 2013, quando então começou um decréscimo pelos anos seguintes. Entretanto, em relação aos idosos, observou-se crescimento importante entre alguns períodos até 2016, havendo redução em 2017.

Tabela 1 - Número de casos de AIDS na região Centro-oeste de 2008 a 2017. Aparecida de Goiânia, 2018.

\begin{tabular}{lcccccc}
\hline Ano & $\mathbf{6 0 - 6 9}$ anos & $\mathbf{7 0 - 7 9}$ anos & $\mathbf{8 0}$ e mais & $<\mathbf{6 0}$ anos & Ignorado & Total \\
\hline 2008 & 87 & 15 & 5 & 2.361 & 0 & 2.468 \\
2009 & 72 & 15 & 3 & 2.450 & 0 & 2.540 \\
2010 & 73 & 19 & 3 & 2.469 & 0 & 2.564 \\
2011 & 91 & 22 & 2 & 2.677 & 0 & 2.792 \\
2012 & 109 & 25 & 6 & 2.832 & 1 & 2.972 \\
2013 & 103 & 35 & 5 & 2.907 & 2 & 3.050 \\
2014 & 106 & 37 & 9 & 2.766 & 1 & 2.918 \\
2015 & 103 & 20 & 5 & 2.600 & 1 & 2.728 \\
2016 & 110 & 33 & 5 & 2.473 & 0 & 2.621 \\
2017 & 49 & 17 & 0 & 1.067 & 0 & 1.133 \\
\hline \multirow{2}{*}{ Total } & $\mathbf{9 0 3}$ & $\mathbf{2 3 8}$ & $\mathbf{4 3}$ & $\mathbf{2 4 . 6 0 2}$ & $\mathbf{5}$ & $\mathbf{2 5 . 7 9 1}$ \\
& $(\mathbf{3 , 5 \% )}$ & $\mathbf{( 0 , 9 2 \% )}$ & $\mathbf{( 0 , 1 7 \% )}$ & $\mathbf{( 9 5 , 4 \% )}$ & $\mathbf{( 0 , 0 1 \% )}$ & $\mathbf{( 1 0 0 \% )}$ \\
\hline
\end{tabular}

Fonte: Dados da pesquisa, 2018.

No tocante às formas de transmissão da AIDS em idosos, observou-se que o maior número de casos, 664 $(56,08 \%)$, deu-se em virtude de relação sexual heterossexual, visto que a via sexual foi a principal responsável pela exposição ao vírus. As categorias concernentes à exposição sexual representam, quando somadas, $59 \%$ dos casos (Tabela 2). 
Tabela 2 - Formas de transmissão de AIDS segundo o ano de diagnóstico na região Centro-oeste de 2008 a 2017. Aparecida de Goiânia, 2018.

\begin{tabular}{lcccccc}
\hline Ano & $\mathbf{6 0 - 6 9}$ anos & $\mathbf{7 0 - 7 9}$ anos & $\mathbf{8 0}$ e mais & $<\mathbf{6 0}$ anos & Ignorado & Total \\
\hline Homossexual & 25 & 2 & 1 & 3.431 & 0 & 3.459 \\
Bissexual & 15 & 3 & 1 & 960 & 0 & 979 \\
Heterossexual & 513 & 132 & 19 & 10.199 & 0 & 10.863 \\
UDI $^{1}$ & 2 & 1 & 1 & 493 & 0 & 497 \\
Hemofílico & 0 & 0 & 0 & 9 & 0 & 9 \\
Transfusão & 1 & 0 & 0 & 3 & 0 & 4 \\
TV2 & 3 & 0 & 0 & 222 & 0 & 225 \\
Ignorado & 344 & 100 & 21 & 9.285 & 5 & 9.750 \\
\hline Total & $\mathbf{9 0 3}$ & $\mathbf{2 3 8}$ & $\mathbf{4 3}$ & $\mathbf{2 4 . 6 0 2}$ & $\mathbf{5}$ & $\mathbf{2 5 . 7 9 1}$ \\
& $\mathbf{( 3 , 5 \% )}$ & $\mathbf{( 0 , 9 2 \% )}$ & $\mathbf{( 0 , 1 7 \% )}$ & $\mathbf{( 9 5 , 4 \% )}$ & $\mathbf{( 0 , 0 1 \% )}$ & $\mathbf{( 1 0 0 \% )}$ \\
\hline
\end{tabular}

1. uso de drogas injetáveis 2. transmissão vertical.

Fonte: Dados da pesquisa, 2018.

Em relação ao sexo, notou-se uma constante variação ao longo dos anos quanto aos dados referentes às categorias masculina e feminina. A predominância do sexo masculino em relação ao feminino se deu numa proporção média de 1,46 casos em homens para cada 1 caso em mulheres (Tabela 3).

Tabela 3 - Casos de AIDS de acordo com o sexo em idosos da região Centro-oeste de 2008 a 2017. Aparecida de Goiânia, 2018.

\begin{tabular}{lccc}
\hline Ano & Masculino & Feminino & Total \\
\hline 2008 & 66 & 41 & 107 \\
2009 & 54 & 36 & 90 \\
2010 & 63 & 32 & 95 \\
2011 & 60 & 55 & 115 \\
2012 & 81 & 59 & 140 \\
2013 & 86 & 57 & 143 \\
2014 & 94 & 58 & 152 \\
2015 & 79 & 49 & 128 \\
2016 & 88 & 60 & 148 \\
2017 & 32 & 34 & 66 \\
\hline Total & $\mathbf{7 0 3}$ & $\mathbf{4 8 1}$ & $\mathbf{1 . 1 8 4}$ \\
& $\mathbf{( 3 , 5 \% )}$ & $\mathbf{( 0 , 9 2 \% )}$ & $\mathbf{( 0 , 1 7 \% )}$ \\
\hline
\end{tabular}

Fonte: Dados da pesquisa, 2018.

\section{DISCUSSÃO}

O número de idosos diagnosticados com AIDS na região brasileira Centro-Oeste, representa 4,59\% do total de casos, sendo o valor absoluto de 1.184 pacientes em um período de 10 anos. Apesar dessa porcentagem parecer baixa, ela propõe uma mudança de paradigma no que concerne as previsões epidemiológicas, fazendo-se relevante o presente estudo. 
De acordo com dados da The Joint United Nations Program on HIV/AIDS (UNAIDS), em 2017 haviam 36,9 milhões de pessoas ao redor do mundo vivendo com HIV/AIDS, sendo 35,1 milhões de adultos (UNAIDS, 2018). Muitos estudos apresentam dados relacionados à visão do idoso como ser assexuado, ou seja, sem vida sexual (CARNEIRO MS, 2013; GARCIA G, et al., 2012; LIMA TC; FREITAS MIP, 2012), no entanto, $39,2 \%$ das pessoas com mais de 60 anos são sexualmente ativas, o que implica deixar de lado o estigma de população idosa assexuada e sendo necessário criar ações em saúde direcionadas a essa faixa etária (OLIVI $\mathrm{M}$, et al., 2008).

Esse fato demonstra quão necessária é a avaliação da prevalência dessa doença em meio aos idosos, e justifica a imprescindibilidade de constantes estudos que evidenciem fatores de risco e possibilitem meios efetivos de prevenção. Contudo, há um hiato bibliográfico com carência de estudos multicêntricos que guiem e sugiram estratégias referentes a esse assunto (DORNELAS NETO J, et al., 2015).

Outro achado relevante foi que o maior número de idosos diagnosticados com AIDS encontra-se no intervalo de 60-69 anos de idade, sendo que o pico de incidência foi no ano de 2016, apresentando 110 casos. Esse número propõe uma dicotomia: o surgimento da terapia antirretroviral, que possibilita maior sobrevida de qualidade aos pacientes, e o aumento de novos casos, que evidencia a adoção de comportamentos de risco por idosos. Dessa forma, torna-se importante valorizar a educação em saúde voltada ao público idoso, com foco nas infecções sexualmente transmissíveis, vias de transmissão das doenças e medidas preventivas corretas (BRITO NMI, et al., 2016).

O aumento de novos casos nos últimos 10 anos possivelmente é consequência de uma maior taxa de detecção da doença após os 60 anos de idade (INELMEN EM et al., 2005). A criação de políticas, melhoria dos serviços de saúde e ampliação da cobertura no estado, como por exemplo a criação de quinze Centros de Testagem e Aconselhamento (CTA), em Goiás, contribuem para esta realidade (SGC, 2019).

É importante ressaltar que existem no Brasil várias políticas centradas no envelhecimento ativo, o que acarreta para esse grupo uma maior qualidade de vida, incluindo o prolongamento da vida sexual. Adicionado aos avanços farmacológicos, com tratamentos hormonais e medicamentos que melhoram o desempenho sexual, observa-se um maior risco de práticas sexuais inseguras. Uma das estratégias dessas políticas é o incentivo à socialização e retomada de vínculos ao envelhecer. Isso se intensifica principalmente com o desenvolvimento de atividades coletivas, turismo e clubes de dança para terceira idade, que possibilitam encontros entre idosos e assim, novos relacionamentos (ANDRADE J, et al., 2017).

É de conhecimento público que, por anos, a AIDS foi tida como uma doença de homens jovens e homossexuais, porém, como demonstrado no estudo, o perfil de acometimento vem sofrendo constantes mudanças. $\mathrm{O}$ aumento de casos de Aids entre idosos no Brasil não é constatado nas demais faixas etárias e constitui-se um desafio encontrar estratégias para enfrentar a situação (SANTOS AFM, ASSIS M, 2011). Porém é importante ressaltar que a vulnerabilidade à doença acontece em todos os ciclos de vida, não sendo adequado designar um perfil único como grupo de risco (MOURA MMS, et al., 2014).

Os diversos setores de saúde e seus profissionais devem se ater e se adequar a essas transformações (AFFELDT AB, et al., 2015) pois, a faixa etária compreendida além dos 60 anos no Brasil permanece sexualmente ativa, ressaltando que o evoluir da idade não elimina ou diminui o desejo sexual, principalmente em homens (BRASIL, 2006; SANTOS AFM, ASSIS M, 2011).

Um importante achado, na população avaliada, foi um maior número de diagnósticos de HIV/AIDS entre os homens, com a maior parte dos casos tendo acontecido por meio de uma relação heterossexual. Esses achados corroboram com os de outros estudos (SILVA MM, et al., 2013; VIANA PAS, et al., 2017).

Os crescentes números de idosos infectados por ISTs demonstram que os atuais meios de educação em saúde ofertados a essa população e as políticas de prevenção adotadas podem não estar sendo efetivos. Muitos profissionais de saúde pecam em estereotipar a população de risco para infecção com o HIV como sendo jovens e homossexuais (DORNELAS NETO J, et al., 2015). 
Tal estereótipo decorre do preceito de que o idoso não possui vida sexual ativa e não se submete a situações de risco, fazendo com que estes permaneçam em situação de risco (CASSÉTTE JB, et al., 2016; ROCHA FCV, et al., 2013).

A prática de sexo desprotegido é indiscutivelmente o principal comportamento de risco mantido pela população idosa devido ao fato de não se considerarem população vulnerável. Com o aumento da idade tende-se a diminuir o uso de preservativos, seja por falta de educação quanto ao uso do método, dificuldade no manuseio, despreocupação com anticoncepção, pela visão do casamento como fator protetor e pela piora no desempenho sexual com uso do preservativo (MELO MC, et al., 2012; DORNELAS NETO J, et al., 2015; ANDRADE J, et al., 2017).

Somam-se a esses fatores, as crescentes exposições a situações de risco relacionadas ao aumento das taxas de divórcio, viuvez, procura de parceiros sexuais na internet e aumento do turismo sexual (NGUYEN N, HOLODNIY M, 2008). Um histórico de outras ISTs durante a vida seria outro fator relevante, mostrando que já existia um comportamento de risco anteriormente (ANDRADE J, et al., 2017).

Apesar de o HIV também ser adquirido por meio de contato com sangue contaminado (transfusão sanguínea e compartilhamento de agulhas e seringas) ou durante a gravidez e amamentação (BRASIL, 2016), neste estudo, um percentual mínimo dos casos esteve ligado às transfusões, reafirmando que o impacto da relação desprotegida é superior a outra forma de exposição ao risco.

Portanto, faz-se necessária a criação de políticas públicas que incluam os idosos como população alvo (AFFELDT AB, et al., 2015), fugindo do enfoque apenas às populações tradicionalmente taxadas como vulneráveis. Ademais, é indispensável que os profissionais sejam incentivados a reconhecer as mudanças comportamentais e epidemiológicas da população e que sejam implementadas intervenções e uma abordagem preventiva nos serviços geriátricos (ANDRADE J, et al., 2017; AFFELDT AB, et al., 2015).

Salienta-se ainda que é imprescindível a capacitação dos profissionais para realizarem uma abordagem da vida sexual nas consultas de rotina, pois isso evidenciará o nível de risco a que o idoso está exposto e levará ao incentivo da adoção de comportamentos seguros (ANDRADE J, et al., 2017). Além disso, é primordial que a família e as instituições frequentadas pelos idosos reconheçam a vulnerabilidade dessa faixa etária para as ISTs e passem a realizar influência positiva, com a divulgação de informações sobre a possibilidade de contrair essas patologias e o estímulo a adoção de hábitos mais saudáveis.

\section{CONCLUSÃO}

O estudo analisou a prevalência de AIDS em idosos do Centro-Oeste entre 2008 e 2017, cujo perfil identificado demonstrou uma predominância de casos na faixa etária entre 60 e 69 anos, forma de infecção mais comum por relação sexual heterossexual, e em indivíduos do sexo masculino. Ressalta-se a importância de atividades de prevenção, diagnóstico e tratamento voltadas à população idosa, que deve ser valorizada em toda sua plenitude e capacidade. Os profissionais de saúde, serviços de ISTs, serviços geriátricos e governos devem estar atentos e conscientes acerca das mudanças de comportamento e perfil epidemiológico da população. Só assim, os idosos terão seus direitos resguardados e serão contemplados na sua integralidade, com ênfase em seu bem-estar e qualidade de vida.

\section{REFERÊNCIAS}

1. AFFELDT AB, SILVEIRA MF, BARCELOS RS. Perfil de pessoas idosas vivendo com HIV/aids em Pelotas, sul do Brasil, 1998 a 2013. Epidemiol. Serv. Saúde, 2015; 24(1): 79-86.

2. ANDRADE J, AYRES JA, ALENCAR RA, DUARTE MTC, PARADA CMGL. Vulnerabilidade de idosos a infecções sexualmente transmissíveis. Acta paul. enferm, 2017; 30(1): 8-15.

3. BRASIL. Ministério da Saúde. Um Guia para se viver mais e melhor. 2006. Disponível em: <http://bvsms.saude.gov.br/bvs/publicacoes/guia_viver_mais_melhor_melhor_2006.pdf >. Acesso em 20 dez. 2018.

4. BRASIL. Ministério da Saúde. Secretaria de Atenção à Saúde. Departamento de Ações Programáticas e Estratégicas. Atenção à saúde da pessoa idosa e envelhecimento. Brasília: 2010. Disponível em URL: http://bvsms.saude.gov.br/bvs/publicacoes/atencao_saude_pessoa_idosa_envelhecimento_v12.pdf 
5. BRASIL. Ministério da Saúde. Departamento de DST, AIDS e Hepatites Virais: AIDS. 2016. O que é HIV. 2013. Brasília. Disponível em: <http://www.aids.gov.br/pagina/o-que-e-hiv>. Acesso em: 11 nov. 2016

6. BRASIL. Ministério da Saúde. Sistemas de Informação de Agravos de Notificação (SINAN). Casos de aids identificados no Brasil. Disponível em: <http://www2.aids.gov.br/cgi/tabcgi.exe?tabnet/br.def>. Acesso em: 04 abr. 2019.

7. BRASIL. Diagnóstico de Idosos. Disponível em: < http://www.aids.gov.br/pagina/diagnostico-de-idosos>. Acesso em 11 jun. 2018.

8. BRITO NMI, ANDRADE SSC, SILVA FMC, FERNANDES MCS, BRITO KKG, OLIVEIRA SHS. Idosos, infecções sexualmente transmissíveis e aids: conhecimentos e percepção de risco. Arquivos Brasileiros de Ciências da Saúde, 2016; 41(3): 140-145.

9. CASSÉTTE JB, SILVA LC, FELÍCIO EEAA, SOARES LA, MORAIS RA, PRADO TS et al. HIV/AIDS among the elderly: stigmas in healthcare work and training. Rev. bras. geriatr. gerontol, 2016; 19(5): 733-744.

10. CARNEIRO MS. Sexuality and aids in the elderly. Rev. Enferm. UFPI, 2013;2(3):67-72.

11. DORNELAS NETO J, NAKAMURA AS, CORTEZ LER, YAMAGUCHI MU. Doenças sexualmente transmissíveis em idosos: uma revisão sistemática. Ciênc. saúde coletiva, 2015; 20(12): 3853-3864.

12. GARCIA G, LIMA LF, SILVA JB et al. Vulnerabilidade dos Idosos frente ao HIV/Aids: Tendências da Produção Cientifica Atual no Brasil. J. bras. doenças sex. transm, 2012; 24(3):183-188.

13. IBGE. Instituto Brasileiro de Geografia e Estatística. Censo Demográfico 2010: Características da população e dos domicílios Resultados do universo $2011 . \quad$ Disponível em: <http://www.ibge.gov.br/home/estatistica/populacao/censo2010/caracteristicas_da_populacao/tabelas_pdf/tab1.pdf>. Acesso em 22 nov. 2018.

14. IBGE. Instituto Brasileiro de Geografia e Estatística. Projeção da população do Brasil por sexo e idade 1980-2050: Revisão 2008-2008. Disponível em: <http://www.ibge.gov.br/home/estatistica/populacao/projecao_da_populacao/2008/projecao.pdf>. Acesso em 22 nov. 2018.

15. INELMEN EM, GASPARINI G, ENZI G. HIV/AIDS in older adults. A case report and literature review. Geriatrics, 2005; 60(9):2630.

16. LAURENTINO NRS, BABOZA D, CHAVES G, BESUTTI J, BERVIAN SA, PORTELLA MR. Namoro na terceira idade e o processo de ser saudável na velhice: recorte ilustrativo de um grupo de mulheres. RBCEH - Revista Brasileira de Ciências do Envelhecimento Humano, 2006; 3(1): 51-63.

17. LAROQUE MF, AFFELDT ÂB, CARDOSO DH, SOUZA GL, SANTANA MG, LANGE C. Sexualidade do idoso: comportamento para a prevenção de DST/AIDS. Rev. Gaúcha Enferm, 2011; 32(4):774-780

18. LIMA TC, FREITAS MIP. Comportamentos em saúde de uma população portadora do HIV/Aids. Rev Bras. Enferm. Brasília, 2012; 65(1): 110-115.

19. LINDAU ST, SCHUMM P, LAUMANN EO, LEVINSON W, O'MUIRCHEARTAIGH CA, WAITE LJ. A study of sexuality and health among older adults in the United States. N Engl J Med, 2007; (24);357:762-74.

20. MASCHIO MBM, BALBINO AP, DE SOUZA PFR, KALINKE LP. Sexualidade na terceira idade: medidas de prevenção para doenças sexualmente transmissíveis e Aids. Revista Gaúcha de Enfermagem, 2011; 32(3): 583-589.

21. MELO MC, PIMENTA AM. Característica epidemiológica da aids na população com mais de 50 anos em Betim e microrregião. R. Enferm. Cent. O. Min., 2012; 2(3): 419-427.

22. MOURA MMS, CARVALHO JFF, GAMA KM, ROCHA FCV. Vulnerability to acquired immune deficiency syndrome in human perception of the elderly. Revista de Enfermagem UFPI, 2014; 3(1):100-106.

23. NGUYEN N, HOLODNIY M. HIV infection in the elderly. Clin Interv Aging, 2008; 3(3):453-72.

24. OLIVI M, SANTANA RG, MATHIAS TAF. Comportamento, conhecimento e percepção de risco sobre doenças sexualmente transmissíveis em um grupo de pessoas com50 anos e mais de idade. Rev. Latino-Am. Enfermagem 2008; 16(4):679-685.

25. ONU. Organização das Nações Unidas. Relatório Mundial de Envelhecimento e Saúde, 2015. Disponível em: <http://apps.who.int/iris/bitstream/handle/10665/186468/WHO_FWC_ALC_15.01_por.pdf;jsessionid=ECDD58147246F1C65A 2DF0C360D815EB? sequence=6>. Acesso em 01 jun. 2018.

26. PIMENTEL CP. HIV na terceira idade: A vulnerabilidade do idoso frente ao vírus da imunodeficiência humana, Anais $\mathrm{V} C \mathrm{CIEH}$, V. 1, 2017, ISSN 2318-0854.

27. ROCHA FCV, FILHO FCF, JÚNIOR JAM, ROSA YRD. Conhecimento dos idosos sobre HIV/AIDS. Revista Interdisciplinar, 2013; 6(2):137-143.

28. SANTOS AFM, ASSIS M. Vulnerabilidade das idosas ao HIV/AIDS: despertar das políticas públicas e profissionais de saúde no contexto da atenção integral: revisão de literatura. Revista Brasileira de Geriatria e Gerontologia, 2011; 14(1): 147-158.

29. SGC. Sistema de Gerenciamento de Conteúdo. Governo de Goiás. Centros de Testagem e Aconselhamento - CTA no estado de Goiás. Disponível em: <http://www.sgc.goias.gov.br/upload/links/arq_516_CTA.pdf>. Acesso em 24 jan. 2019.

30. SILVA MM, VASCONCELOS ALR, RIBEIRO LKNP. Caracterização epidemiológica dos casos de AIDS em pessoas com 60 anos ou mais, Pernambuco, Brasil, 1998 a 2008. Cad. Saúde Pública, 2013; 29(10): 2131-2135.

31. UNAIDS. The Joint United Nations Program on HIV/AIDS. Estatísticas. Disponível em: < https://unaids.org.br/estatisticas/>. Acesso em 10 dez. 2018.

32. VIANA PAS et al. Aspectos epidemiológicos, clínicos e evolutivos da aids em idosos no norte do Ceará. SANARE, 2017; $16(2): 31-36$. 